\title{
A POLÍTICA MUNICIPAL DE SAÚDE EM DADOS: A EXPERIÊNCIA DA PESQUISA MUNICIPAL UNIFICADA - PMU 1992, 1995 E 1997 DA FUNDAÇÃO SEADE
}

\author{
Zilda Pereira da Silva* \\ Irineu Francisco Barreto $\mathrm{Jr}^{\star \star}$

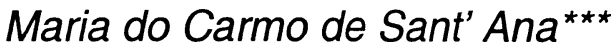

RESUMO: A produção e a disseminação de informações municipais vêm assumindo papel cada vez mais estratégico enquanto instrumento de gerenciamento dos sistemas municipais de saúde e de subsiadiador do diálogo entre o poder central, os setores estaduais e a prefeitura municipal. Com o objetivo de disponibilizar e divulgar informações que procuram valorizar o SUS municipal, foi feita uma pesquisa nos 645 municípios paulistas, cujos resultados foram sistematizados em produto eletrônico com 11 temas. A divulgação de um painel de informações revela que os municípios têm-se adequado aos princípios do SUS. Os resultados também indicam que a necessidade da informação como ferramenta de gestão vem envolvendo, cada vez mais, os responsáveis pelas políticas de saúde. As informações coletadas e disponibilizadas no tema Saúde suprem lacunas de pesquisas primárias que não abordam os 645 municípios do Estado; estas informações e as dos demais temas possibilitam o desenho de um panorama intersetorial de cada um dos municípios.

PALAVRAS-CHAVE: pesquisa municipal unificada, sistemas municipais de saúde, produto eletrônico, gestão, Sistema Único de Saúde

* Sociológa, Coordenadora do Setor de informações de Saúde da Fundação SEADE

** Sociólogo, analista da Fundação SEADE

*** Historiadora, analista da Fundação SEADE 


\section{APRESENTAÇÃO}

A produção de informações municipais vem assumindo papel cada vez mais estratégico enquanto instrumento de gerenciamento dos sistemas municipais de saúde e de subsidiador do diálogo entre o poder central, os gestores estaduais e a prefeitura municipal.

A Pesquisa Municipal Unificada - PMU é um dos projetos mais tradicionais da Fundação Seade ${ }^{1}$, em que se investigam a capacidade organizacional, administrativa e financeira das Prefeituras Municipais, além da realidade socioeconômica, cultural e urbana municipal, através de levantamento cujos resultados possibilitaram a construção do aplicativo Pesquisa Municipal Unificada - PMU 1992, 1995 e 1997, com acesso a uma radiografia dos 645 municípios, por meio de 724 variáveis.(FUNDAÇÃO SEADE, 1999).

Das 204 variáveis do tema Saúde, optou-se, neste estudo, por se organizar um painel de dados, proceder à geração de indicadores de gestão e a análises específicas que se propõem a investigar a Política e Administração da Saúde — através da implantação ou não dos dispositivos legais de participação da comunidade e o controle social sobre a formulação, acompanhamento e avaliação do Sistema Único de Saúde Municipal e da utilização ou não da informação como ferramenta de gestão na condução, avaliação e controle das políticas de saúde.

\section{JUSTIFICATIVA}

A implantação do Sistema Único de Saúde - SUS, materializado pela Constituição de 1988 e regulamentado em 1990, vem exigindo diferentes transformações na cultura organizacional do setor Saúde, envolvendo governos, autoridades, gestores, profissionais e técnicos de saúde, organizações, sistemas sociais, lideranças comunitárias e população em geral, atores fundamentais do planejamento, acompanhamento, avaliação, controle e auditoria desse novo modelo de gestão que tem no município seu principal sujeito.

Essa nova realidade faz da informação em saúde um instrumento de apoio decisivo para o conhecimento socioeconômico, demográfico e epidemiológico local, assim como para se proceder a uma visualização integral do contexto regional e nacional em que se insere.

lÓrgão vinculado à Secretaria de Economia e Planejamento do Estado de São Paulo 
Os resultados da Pesquisa Municipal Unificada - PMU, ora analisados, têm como fonte de informações as próprias Prefeituras, estão consolidados num produto eletrônico de fácil operacionalização e recuperação de dados e podem ser vistos como uma das respostas às necessidades instrumentais para o acompanhamento da política municipal de saúde.

\section{OBJETIVOS}

A criação do Sistema Único de Saúde delegou aos municípios uma série de atribuições, anteriormente a cargo da União e dos Estados, que perpassa pela autonomia na elaboração da política municipal de saúde, alcançando o gerenciamento da rede básica de saúde, a gestão dos recursos humanos e o desenvolvimento de ações de vigilância e promoção de saúde individual e coletiva.

O aplicativo Pesquisa Municipal Unificada - PMU 1992, 1995 e 1997 (FUNDAÇÃO SEADE, 1999) tem o objetivo de manter atualizado um banco de dados sobre os 645 municípios paulistas, que possibilite a construção de indicadores de gestão e contribua para a concepção do calendário de prioridades da intervenção pública, para a formulação de planos, estratégias de gerenciamento e avaliação das políticas regionais e municipais e para a análise dos programas e das ações municipais em relação às demandas sociais, em municípios de diferentes portes.

O tema Saúde, em específico, investigou a dinâmica do processo de municipalização das políticas deste setor, no Estado, em direção à descentralização político-administrativa. Atualizou informações já obtidas, nas versões da PMU 1992 e 1995, complementadas com questões acerca das novas diretrizes do Ministério da Saúde para a gestão municipal do SUS.

\section{METODOLOGIA}

Frente à heterogeneidade do conjunto dos 645 municípios paulistas, a configuração dos sistemas municipais de saúde também deve ser marcada por uma diferenciação na capacidade e na disponibilidade política das prefeituras e das comunidades em assumirem as novas atribuições. A Pesquisa Municipal Unificada é uma dos únicos trabalhos de investigação que tem nas prefeituras municipais fontes primárias de dados, o que permite conhecer essa nova realidade. Levada a campo para todos os municípios do Estado, em 1997 alcançou o retorno expressivo de $96,4 \%$ de respostas. 
A estratégia para alcance desse universo foi executada em diferentes etapas. Num primeiro momento, as equipes técnicas da Fundação Seade trabalharam no aperfeiçoamento dos questionários de coleta de dados, aplicados em 1992 e 1995, adaptando-os às alterações dos rumos das políticas setoriais, como da saúde e da educação, e discutindo seus conteúdos com técnicos envolvidos na gestão e no acompanhamento destas políticas. Em seguida, foram desenvolvidos os programas de entrada de dados, específicos para cada questionário, com as rotinas de consistência previstas pelas equipes técnicas quando da elaboração desses instrumentos, o que resultou em maior agilidade nos procedimentos operacionais e no controle da qualidade das informações obtidas.

Ressalte-se que foi solicitado a cada uma das Prefeituras que indicassem um coordenador local da pesquisa, responsável pela distribuição dos questionários entre os diferentes setores da administração municipal e também pela devolutiva deste material ao pesquisador encarregado. A remessa dos questionários foi feita por Sedex e o recolhimento - em cada um dos municípios - por pesquisadores devidamente capacitados através de treinamento de 40 horas, dividido em temas e monitorado pelo corpo técnico das áreas a serem investigadas.

Simultaneamente à distribuição dos questionários foram desenhados instrumentos para o trabalho de campo - guia do coordenador, manual do pesquisador, manual de crítica e consistência e relatório para uso no plantão técnico de resolução de problemas por telefone.

A estratégia de recolher o questionário in loco teve como objetivo garantir a qualidade e a fidedignidade das informações coletadas, cabendo aos pesquisadores realizar conferência e revisão das mesmas, obedecendo a critérios estabelecidos através de parâmetros de crítica - informações obtidas nas PMUs de 1992 e 1995 - que serviram como determinantes para as respostas de 1997.

Uma vez recolhido o conjunto de questionários em cada município, uma equipe devidamente treinada de supervisores procedeu a uma análise das informações coletadas, avalizando ou não a sua consistência, para posterior digitação. Verificada alguma inconsistência nas respostas obtidas, uma equipe foi encarregada de contatar os municípios e validar ou não estas informações.

Com os resultados obtidos construiu-se uma base de dados de saúde composta por 204 variáveis que integram um produto eletrônico constituído por 10 termas: Estrutura Administrativa; Política Urbana; Habitação; Transportes; Limpeza Pública; Saneamento Básico; Saúde; Educação; Cultura e Esportes; e Finanças Públicas, incorporando também 
o dado de População Total, estimado pelo Seade, possibilitando o desenho de um panorama intersetorial de cada um dos municípios do Estado.

O questionário de Saúde, dividido por subtemas - Política e Administração, Serviço de Vigilância, Recursos Humanos, Transferência de Pacientes, Programas e Ações de Saúde e Informatização — foi respondido por 615 prefeituras, perfazendo $95 \%$ dos municípios paulistas.

A instalação de 73 novos municípios no Estado de São Paulo, entre 1993 e 1997 e a dinâmica da política nacional, regional e municipal de saúde, nos anos 90 , foram responsáveis pela limitação imposta à construção de séries históricas das informações analisadas. Não se trabalharam, portanto, neste estudo, os dados de 1992 e algumas variáveis que não são passíveis de comparação no período 1995-1997, uma vez que foram inseridas à pesquisa neste último ano.

Tendo em vista as diversidades locais — porte físico, territorial e dinâmica econômica, social e urbana - os municípios foram classificados em seis diferentes estratos populacionais:

- municípios com até 10.000 habitantes;

- municípios entre 10.001 e 20.000 habitantes;

- municípios entre 20.001 e 50.000 habitantes;

- municípios entre 50.001 e 100.000 habitantes;

· municípios entre 100.001 e 200.000 habitantes;

- municípios acima de 200.001 habitantes (exceto a Capital);

- Capital ${ }^{2}$.

\section{DISCUTINDO OS RESULTADOS DA PESQUISA MUNICIPAL UNIFICADA}

\section{Política e Administração da Saúde}

A Pesquisa Municipal Unificada - PMU permite que se visualizem importantes mudanças ocorridas na gestão da política da saúde e as informações, ora analisadas,

2 Nos dados comparativos entre 1995 e 1997 não está incluído o município de São Paulo, por não ter respondido a PMU 1995. 
procuram valorizar o SUS Municipal, destacando-se aquelas variáveis que revelam a participação da sociedade civil organizada e o controle social, preconizados pelas diretrizes do SUS, e uma transformação na prática gerencial, administrativa e estratégica na condução das políticas de saúde por parte das prefeituras, que consiste na criação, manutenção e uso de bancos de dados municipais.

\section{Controle Social}

Os conselhos municipais de saúde foram criados com o objetivo de atuar na formulação e no controle da política de saúde, incluídos os aspectos econômicos e financeiros (Lei 8.142/90). Enquanto princípio, o controle social no SUS já constava na Constituição de 1988 e foi reafirmado nas Normas Operacionais Básicas 1993 e 1996 do Ministério da Saúde. Segundo CARVALHO (1995), os conselhos de saúde emergiram no cenário político sanitário brasileiro a partir das reformulações setoriais que deram origem ao Sistema Único de Saúde e integram, concretamente desde 1990, a configuração institucional desse sistema, cabendo-Ihes a missão de garantir o cumprimento do princípio constitucional da "participação da comunidade" e assegurar o controle social sobre as ações e serviços de saúde.

Através dos dados da PMU, verifica-se que os municípios, após uma década da Constituição que estabeleceu os princípios da descentralização e da autonomia, em sua quase totalidade criaram conselhos municipais de saúde (92\%), sendo que, destes, $54 \%$ possuem composição paritária entre os representantes dos usuários e dos demais segmentos $^{3}$. Observando-se a Tabela 1, percebe-se a perspectiva do porte populacional não foi uma variável determinante para a existência ou não de um conselho de saúde:

3 Os demais segmentos consistem nos representantes dos prestadores de serviços, dos governos e dos profissionais de saúde. 
Tabela 1 - Municípios que Possuem ou Não Conselho Municipal de Saúde, Segundo os Estratos Populacionais Estado de São Paulo 1997

\begin{tabular}{|c|c|c|c|c|c|c|}
\hline \multirow{3}{*}{$\begin{array}{l}\text { Estratos } \\
\text { Populacionais }\end{array}$} & \multicolumn{6}{|c|}{ Municipios que Possuem ou Não Conselho Municipal de Saúde } \\
\hline & \multicolumn{2}{|l|}{ Sim } & \multicolumn{2}{|l|}{ Não } & \multicolumn{2}{|c|}{ Total } \\
\hline & $\begin{array}{l}\text { Números } \\
\text { Absolutos }\end{array}$ & $\%$ & $\begin{array}{l}\text { Números } \\
\text { Absolutos }\end{array}$ & $\%$ & $\begin{array}{l}\text { Números } \\
\text { Absolutos }\end{array}$ & $\%$ \\
\hline Total & 595 & 96,75 & 20 & 3,25 & 615 & 100,00 \\
\hline Até 10.000 & 276 & 95,50 & 13 & 4,50 & 289 & 100,00 \\
\hline De 10.001 a 20.000 & 106 & 95,50 & 5 & 4,50 & 111 & 100,00 \\
\hline De 20.001 a 50.000 & 110 & 99,10 & 1 & 0,90 & 111 & 100,00 \\
\hline De 50.001 a 100.000 & 47 & 100,00 & - & - & 47 & 100,00 \\
\hline De 100.001 a 200.000 & 33 & 100,00 & - & - & 33 & 100,00 \\
\hline Acima de 200.000 & 23 & 100,00 & - & - & 23 & 100,00 \\
\hline Capital & - & - & 1 & 100,00 & 1 & 100,00 \\
\hline
\end{tabular}

Fonte: Fundação Seade. Pesquisa Municipal Unificada - PMU 1992, 1995 e 1997.

Quanto à Capital do Estado, a atual gestão da Secretaria Municipal de Saúde informou que, o conselho municipal de saúde foi criado por lei apenas no ano de 1998, um ano após o investigado pela PMU, desconsiderando assim a existência do conselho de saúde que vigorava no Município de São Paulo há quase uma década e que havai sido criado por uma portaria. Este é apenas um dos vários aspectos que envolvem a polêmica gestão na área de saúde da Prefeitura de São Paulo.

Com relação aos representantes de usuários nos conselhos, observa-se que diferentes segmentos da sociedade civil organizada estão neles representados: associações de moradores (em $64 \%$ dos conselhos); movimentos comunitários (54\%); organizações religiosas (53\%); sindicatos (48\%); associações de portadores de deficiências e/ou doenças crônicas (25\%) e associações de defesa do consumidor (5\%).

As informações permitem, também, que se conheça o ano de implantação dos conselhos municipais de saúde, constatando-se que mais da metade desses conselhos 
(53\%) foram implantados no ano de 1991, sob a égide da Lei Orgânica da Saúde (8.080/ 90). A instalação dos demais distribui-se entre os anos de 1992 (4\%); 1993 (18\%); 1994 (7\%); 1995 (3\%); 1996 (1\%); e 1997 (15\%), percebendo-se que as edições das Normas Operacionais Básicas - NOBs, do Ministério da Saúde, em 1993 e 1996 - que passou a vigorar no início de 1998 - reafirmaram a importância dos mecanismos de participação social.

Um indicador da efetividade dos conselhos foi o estudo da periodicidade de suas reuniões, também investigada pela PMU. Observou-se que a periodicidade mensal foi a mais citada (58\% dos casos), distribuindo-se as demais respostas da seguinte forma: periodicidade anual (1\%); semestral (2\%); quadrimestral (1\%); trimestral $(9 \%)$; bimestral $(18 \%)$; quinzenal $(2 \%)$; semanal $(0,3 \%)$; e eventual $(6 \%)^{4}$. Causa certa preocupação o fato de que percentual de conselhos que não realizavam pelo menos uma reunião mensal, somado àqueles que se reuniam eventualmente, estivesse no patamar de $37 \%$, mais de um terço do total de municípios investigados.

A configuração da política e administração da saúde contemplou também outro importante aspecto do controle social na gestão dos sistemas locais, isto é, a realização de conferências municipais de saúde. Assim como a existência do conselho, a realização de conferência está prevista na Lei 8.142 de 1990 e é considerada instância colegiada do SUS. Esta lei define que a conferência deve reunir-se a cada quatro anos, convocada pelo Poder Executivo, ou, extraordinariamente convocada pelo mesmo Poder Executivo ou pelo conselho, para avaliar a situação da saúde e propor as diretrizes para a reformulação da política setorial no nível local (MINISTÉRIO DA SAÚDE, 1993).

Os dados revelaram, no entanto, que até 1997, apenas $28 \%$ dos municípios haviam realizado conferência, informação que pode ser observada também segundo os estratos populacionais. Nesta perspectiva, observa-se que a variável porte foi determinante:

$45 \%$ dos municípios não responderam esse item do questionário. 
Tabela 2 - Número de Municipios que Realizaram ou Não Conferência Municipal de Saúde, Segundo Estratos Populacionais Estado de São Paulo 1997

\begin{tabular}{|c|c|c|c|c|c|c|c|}
\hline \multirow{3}{*}{$\begin{array}{l}\text { Estratos } \\
\text { Populacionais }\end{array}$} & \multicolumn{7}{|c|}{ Municipios que Realizaram ou NãoConferência Municipal de Saúde } \\
\hline & \multicolumn{3}{|c|}{ Sim } & \multicolumn{2}{|c|}{ Não } & \multicolumn{2}{|c|}{ Total } \\
\hline & $\begin{array}{l}\text { Nún } \\
\text { Abs }\end{array}$ & $\begin{array}{l}\text { neros } \\
\text { olutos }\end{array}$ & $\%$ & $\begin{array}{l}\text { Números } \\
\text { Absolutos }\end{array}$ & $\%$ & $\begin{array}{l}\text { Números } \\
\text { Absolutos }\end{array}$ & $\%$ \\
\hline Total & & 183 & 29,76 & 432 & 70,24 & 615 & 100,00 \\
\hline \multicolumn{2}{|l|}{ Até 10.000} & 42 & 14,53 & 247 & 85,47 & 289 & 100,00 \\
\hline \multicolumn{2}{|l|}{ De 10.001 a 20.000} & 30 & 27,03 & 81 & 72,97 & 111 & 100,00 \\
\hline \multicolumn{2}{|l|}{ De 20.001 a 50.000} & 48 & 43,24 & 63 & 56,76 & 111 & 100,00 \\
\hline \multicolumn{2}{|c|}{ De 50.001 a 100.000} & 22 & 46,81 & 25 & 53,19 & 47 & 100,00 \\
\hline \multicolumn{2}{|c|}{ De 100.001 a 200.000} & 22 & 66,67 & 11 & 33,33 & 33 & 100,00 \\
\hline \multicolumn{2}{|l|}{ Acima de 200.000} & 18 & 78,26 & 5 & 21,74 & 23 & 100,00 \\
\hline \multicolumn{2}{|l|}{ Capital } & 1 & 100,00 & - & - & 1 & 100,00 \\
\hline
\end{tabular}

Fonte: Fundação Seade. Pesquisa Municipal Unificada - PMU 1992, 1995 e 1997.

Nota: Exceto 30 municípios que não responderam o questionário de Saúde da PMU97, do universo de 645 existentes no Estado em 1997.

Dessa forma, percebe-se que este mecanismo de controle social do SUS foi implementado nos municípios com as populações maiores. Certamente, este fenômeno está associado à disponibilidade de infra-estrutura e recursos necessários para a realização de uma conferência municipal de saúde, sem que se levem em consideração vontade política, grau de organização da sociedade, de maturidade e de compromisso dos conselhos, variáveis incomensuráveis neste tipo de levantamento.

\section{BANCO DE DADOS DE SAÚDE}

A informação em saúde (CARVALHO \& EDUARDO, 1998) deve ser entendida como um instrumento de apoio decisório para o conhecimento da realidade socioeconômica, demográfica e epidemiológica, para o planejamento, gestão, organização e avaliação nos vários níveis que constituem o SUS e, pode-se dizer, há um consenso de que não é possível 
A política Municipal de Saúde em dados: a experiência da pesquisa municipal unificada da Fundação SEADE

exercer gerência em nenhum setor se não houver um sistema de apoio à decisão que se sustente na informação.

A utilização da informação como ferramenta de gestão vem envolvendo cada vez mais os responsáveis pelas políticas de saúde. Esse fenômeno transcende a questão técnica e coloca no cerne do debate a implementação de novos modelos de gerência e de gestão dos sistemas locais. Os dados sobre a existência e a utilização de banco de dados de saúde municipais permitem, numa primeira abordagem, a compreensão desta nova configuração dos sistemas municipais de saúde, comprometida com o aumento da eficiência do sistema e com a geração da eqüidade (CARVALHO \& EDUARDO, 1998).

A existência do banco de dados de saúde foi apontada por $79 \%$ dos municípios, com incremento de 8 pontos percentuais em relação ao ano de 1995. Estes resultados podem estar indicando que a informação, como ferramenta de gestão, vem envolvendo cada vez mais os responsáveis pelas políticas de saúde dos municípios, em todos os estratos populacionais estudados, o que é corroborado pelos $17 \%$ dos municípios que promoveram cursos de capacitação e/ou treinamento de seus recursos humanos na área de produção, análise e disseminação de dados e informações em saúde, com vistas a uma maior familiarização e aproveitamento dessas técnicas.

Quanto aos tipos de dados que compõem estes bancos, foram mais freqüentes os que se referem a: produção ambulatorial (75\%); doenças de notificação compulsória (74\%); cadastro de unidades de saúde (72\%); atendimento a gestantes $(72 \%)$; número de consultas de pré-natal $(71 \%)$; número médio de consultas de pré-natal $(65 \%)$ e número de gestantes inscritas no pré-natal (64\%).

É possivel ainda que se conheçam os serviços gerenciados pelas prefeituras que utilizaram informações do banco de dados de saúde municipal: gerência da vigilância à saúde da mulher (76\%); vigilância ao recém-nascido (61\%); e vigilância à saúde do trabalhador (20\%).

Dessa maneira, os dados da PMU permitem um dimensionamento preliminar da utilização dessa importante ferramenta na gestão municipal da saúde, que são os bancos de dados, transformados em informação para gerenciamento.

\section{CONCLUSÕES}

Os indicadores analisados trazem à tona a resposta positiva dada pelas prefeituras municipais e pela população do Estado de São Paulo aos preceitos constitucionais e legais 
que fundamentam e regulamentam o Sistema Único de Saúde quanto à participação e ao controle da sociedade sobre a sua implementação.

Verifica-se a existência de conselhos de saúde na quase totalidade dos municípios paulistas, com representatividade dos usuários nos diferentes setores da sociedade civil organizada - associações de moradores, movimentos comunitários, organizações religiosas, sindicatos, associações de portadores de deficiências e/ou doenças crônicas e associações de defesa do consumidor, dentre os mais freqüentes.

As informações demonstram também que, embora $97 \%$ dos municípios tivessem conselhos implantados, mais de um terço deles não sistematiza a periodicidade de suas reuniões e que apenas 28\% já tinham realizado conferência municipal de saúde, até 1997.

A existência e a utilização da informação de saúde municipal podem ser consideradas fenômeno também quase que consagrado, uma vez que essas variáveis foram apontadas com considerável freqüência.

A realização de cursos de capacitação e/ou treinamento de recursos humanos na área de produção, análise e disseminação de dados e informações de saúde obteve resposta positiva em $17 \%$ dos municípios investigados.

As informações disponibilizadas certamente vêm suprir lacunas de pesquisas primárias que não abordam os 645 municípios do Estado e são inovadoras por servirem à geração de indicadores de gestão. Acreditamos também que podem ser um passo a mais em direção ao fortalecimento da idéia de tornar o uso da informação parte da cultura organizacional das instituições governamentais e ferramenta de implementação das habilidades daqueles que ocupam postos gerenciais, técnicos, acadêmicos, administrativos e estudiosos do assunto.

\section{REFERÊNCIAS BIBLIOGRÁFICAS}

CARVALHO, A.O. \& EDUARDO, M.B.P. Sistemas de informação em saúde para municípios. Série Saúde e Cidadania para Gestores Municipais de Serviços de Saúde, $n^{0 .}$ 6. São Paulo, Faculdade de Saúde Pública da Universidade de São Paulo, 1998.

CARVALHO, A.I. Conselhos de saúde no Brasil. Série Estudos Política, Planejamento e Gestão em Saúde n ${ }^{\circ}$ 3. Rio de Janeiro, Fiocruz, 1995.

FUNDAÇÃo SEADE Pesquisa Municipal Unificada - 1992, 1995 e 1997. São Paulo, Fundação Seade, 1999 (edição eletrônica). 
MINISTÉRIO DA SAÜDE. Conselho de Saúde: guia de referência para a sua criação e organização. Brasília, 1993.

\section{THE MUNICIPAL HEALTH POLICY IN DATA: THE UNIFIED MUNICIPAL RESEARCH EXPERIENCE - PMU 1992, 1995 AND 1997}

SUMMARY: Production and dissemination of municipal information have played an increasingly strategic role as management tools of municipal health systems and dialogue subsidizer among federal authorities, state sectors and municipal authorities. Research was carried out in 645 cities in the State of São Paulo and the results systematized in an electronic product with 11 topics. Its purpose was to make information available and disseminate information that values the municipal SUS. The promotion of an information board reveals that the cities have adjusted to SUS principles. The results also indicate that the need to have information as a management tool is increasingly involving those responsible for health policies. Information gathered and conveyed in the topic. Health topic fills gaps of primary research that did not cover the 645 cities of the State. This information and data on other topics enable the design of an intersectorial panorama of each city.

KEY WORDS: unified municipal research, municipal health system, electronic product, management, Unified Health System 